
\title{
ZS Research Suare \\ The Influence of SSO on the Optimization Result of Nasopharynx Carcinoma Plan
}

\section{Xiaolong HUA}

Xinghua People's Hospital

Jianhe YU

Xinghua People's Hospital

Lu WANG ( $\sim$ WL_AsCa@163.com )

Anshan Cancer Hospital https://orcid.org/0000-0002-2576-2605

Jiaqi DAI

Elekta Instrument (Shanghai) Ltd

\section{Qun REN}

Xinghua People's Hospital

\section{Li CHEN}

Anshan Cancer Hospital

Chuanjun SONG

Xinghua People's Hospital

\section{Yanshu MOU}

Anshan Cancer Hospital

\section{Wenjing XU}

Xinghua People's Hospital

Hongxia XU

Xinghua People's Hospital

\section{Research}

Keywords: SSO, XVMC, VMAT, NPC

Posted Date: June 28th, 2021

DOl: https://doi.org/10.21203/rs.3.rs-635874/v1

License: @ (1) This work is licensed under a Creative Commons Attribution 4.0 International License. Read Full License 


\section{Abstract}

\section{Purpose}

To study the influence of Monaco 5.4 treatment planning system (TPS) on the dosimetry of radiotherapy for nasopharynx carcinoma (NPC) under the condition of different segment shape optimization (SSO) times.

\section{Methods}

Fifteen patients with $\mathrm{T}_{3-4} \mathrm{~N}_{0-2} \mathrm{M}_{0}$ stage nasopharyngeal carcinoma were enrolled, and each case was designed with SSO of 3, 5, 7 and 10 times respectively. The dose results of the target area and the major organs at risk (OAR) were statistically analyzed by DVH statistics; moreover, the isodose lines of 70Gy, 60Gy and 54Gy were intercepted at the same plane in the transverse, coronal and sagittal views and the segment shapes were compared at the angle of $30^{\circ}, 120^{\circ}, 240^{\circ}$ and $330^{\circ}$ in beam eye view (BEV); In addition, optimization time (OT), delivery time (DT), segments\# and MU\# were obtained and analyzed by optimization console; the plans were verified and analyzed by using ArcCheck phantom.

\section{Results}

For target area $\mathrm{D}_{2}$, the results of the SSO7 group and the SSO10 group were similar and both better than those of SSO3 and SSO5 groups, and the $\mathrm{D}_{2}$ results of the $\mathrm{SSO} 3$ group were notable higher than those of the other three groups; for the major OARs, the results of the maximum dose of spinal cord, brain stem, and lens and the mean dose and $V_{30}$ of parotid glands showed the same trend. It showed that SSO7 and SSO10 share similar dose results, too which are notable better than the similar dose results shared by SSO3 and SSO5; in the dose deprogram distribution of 70Gy, 60Gy and 54Gy, partial 70Gy dose spillover occurred in both groups SSO3 and $\mathrm{SSO} 5$ and it was more obvious in group SSO3. While there was a no significant dose spillover in group SSO7 and group SSO10; in the sub-field alignment comparison under the same angle, the alignment became more complicated and the sub-fields were smaller as the number of SSO increased; the results of segment\#, MU\# and plan delivery time between different SSO groups were slightly different, while the plan optimization time changed significantly. The difference between group SSO3 and group SSO10 was more than 500s; the results were compared in ArcCheck, there was no significant difference between the groups.

\section{Conclusions}

The user-defined SSO function of Monaco 5.4 TPS effectively balances the relationship between plan design efficiency and plan quality. When SSO is 7, it is better value for efficiency and quality in clinical radiotherapy for nasopharyngeal carcinoma.

\section{Background}

Nasopharyngeal carcinoma is known to be endemic in some regions of the world, especially the Southern Chinese. The radiation therapy is the standard radical treatment for NPC because of its high radio sensitivity and difficulty removing by surgery ${ }^{[1-3]}$. Monaco of Elekta is one of the most outstanding treatment planning system (TPS) for radiation therapy, and its X-ray voxel Monte Carlo (XVMC) algorithm is recognized as one of the maturest commercial photon Monte Carlo algorithms at present ${ }^{[4]}$. XVMC is the core algorithm, it tries to 
obtain the most suitable shape of the sub-fields for the plan through several times of segments shape optimization (SSO), and each SSO contains several inter loops and outer loops. In retrospect of 100 cases of nasopharyngeal carcinoma from 2019 to 2020, we interpreted the optimization process by the optimization console function, and we found that Monaco TPS 5.1 version was fixed for 5 times of SSO. However, planning for nasopharyngeal carcinoma is one of the most complex types of radiotherapy plan design ${ }^{[5,6,7,8]}$. The nasopharynx is adjacent to important normal tissues such as brainstem, spinal cord, lens and parotid glands, in the meanwhile, the target area is usually large and irregular $[9,10,11]$.

Therefore, how to design higher quality plan more efficiently has become an important research topic. In Monaco TPS 5.4 version, the optimization of SSO was upgraded from fixed times to autonomous definition, which led to whether different SSO settings would have an impact on the optimization results. In this paper, the differences of different SSO plans were compared, so as providing a reference for clinical design of NPC plans.

\section{Materials And Methods}

\section{Data}

Case selection and plan design: 15 patients with $\mathrm{T}_{3-4} \mathrm{~N}_{0-2} \mathrm{M}_{0}$ NPC were enrolled in the study. Axial cinematic scanning was performed in free breathing condition, the CT rotation time was 0.8 seconds, the axial thickness was $2.5 \mathrm{~mm}$, the voltage was $120 \mathrm{KV}$ and the current was $350 \mathrm{mAs}$. All images were uploaded to Monaco 5.4 TPS, and the target area was contoured by radiotherapy physicians with more than 5 years working experience in accordance with NCCN2020 NPC treatment guidelines, and the target area was reviewed by chief physicians with more than 10 years working experience. The plan was designed by a senior clinical physicist with more than 10 years of Monaco TPS experience.

\section{Plan Design}

Dual full volumetric modulated arc therapy (VMAT) can generate precise conformal dose distribution and was used in all the plans ${ }^{[12]}$. Under condition of the functions and TPS parameter settings remain unchanged, XVMC optimization was performed for 3, 5, 7 and 10 times of SSO; a fraction dose of 2Gy, a fraction of 30, and the dose constraint of OARs was carried out according to QUANTEC (Quantitative Analysis of Normal Tissue Effects in the Clinic) report. The dose results, conformity index $(\mathrm{Cl})$ and homogeneity index $(\mathrm{HI})$ of target area and major OARs of different SSO plans were statistically analyzed by DVH statistics ${ }^{[13]}$. The multi-leaf collimator (MLC) shapes were intercepted and compared at $30^{\circ}, 120^{\circ}, 240^{\circ}$ and $330^{\circ}$ in the BEV interface.

The dose structures of PGTV 70 and PTV 60 target areas were established, and the prescription dose coverage rates were calculated by Formula (1). Since 54Gy covers the structure of PTV60 and PTV54 target areas at the same time, the dose structure of 54Gy was cut into two dose structures, PTV 60 - 54Gy and PTV $54-54$ Gy, at the interface layer of the two target areas, for calculation and evaluation respectively; transverse, coronal and sagittal dose nephograms of 70Gy, 60Gy and 54Gy were intercepted and compared in the same image layer; OT, DT, segments\# and MU\# were obtained and analyzed by optimization console. The plan test environment is a standard HP Z8 desktop server equipped by Elekta. 
$\mathrm{R}=\frac{V_{G y}}{V_{\text {Target }}} \times 100 \%$

Where $\mathrm{R}(\%)$ is the prescription dose coverage rate, $\mathrm{V}_{\mathrm{Gy}}\left(\mathrm{cm}^{3}\right)$ is the volume of the assessed dose coverage, and $\mathrm{V}_{\text {Target }}\left(\mathrm{cm}^{3}\right)$ is the volume of the assessed target area.

\section{Plan Verification}

60 cases in 15 groups were imported into ArcCheck for plan verification, and the results of plan verification were statistically analyzed.

\section{Statistical analysis methods}

Data was collected by SPSS 22.0, and paired t-test was performed on the dose results, $\mathrm{P}<0.05$ was considered statistically significant. In Monaco 5.1 TPS, SSO5 was the default value, so the results of other groups were matched with those of SSO5 group by paired t-test.

\section{Results}

\section{Comparison of dose results between groups}

The target dose results (Table 1) and the major OARs dose results data (Table 2) of 60 cases in 15 groups with the same parameters except SSO were read by Monaco TPS DVH statistics function. Under the calculation method of target area priority, $\mathrm{PGTV}_{70}$ and $\mathrm{PTV}_{60}$ reached similar results in the percentage of prescription dose coverage, while PTV $V_{54}$ had significant difference; in the dose statistics of $D_{2}$ and $D_{\text {mean }}$ in each target area, the SSO3 result was significantly higher than that of other groups; in comparison of $\mathrm{HI}$ and $\mathrm{Cl}$ results, $\mathrm{Cl}$ results showed significant difference, and $\mathrm{HI}$ results showed different performance in different target areas. In the statistical analysis of the major OARs dose results, In the statistical analysis of the major OARs dose results, the maximum dose and volume dose optimization results gradually decreased with the increase in the number of SSOs, while the results of the SSO7 group and the SSO10 group were similar.

Table 2. The effect of diffent SSO on OARs dose results\&区 


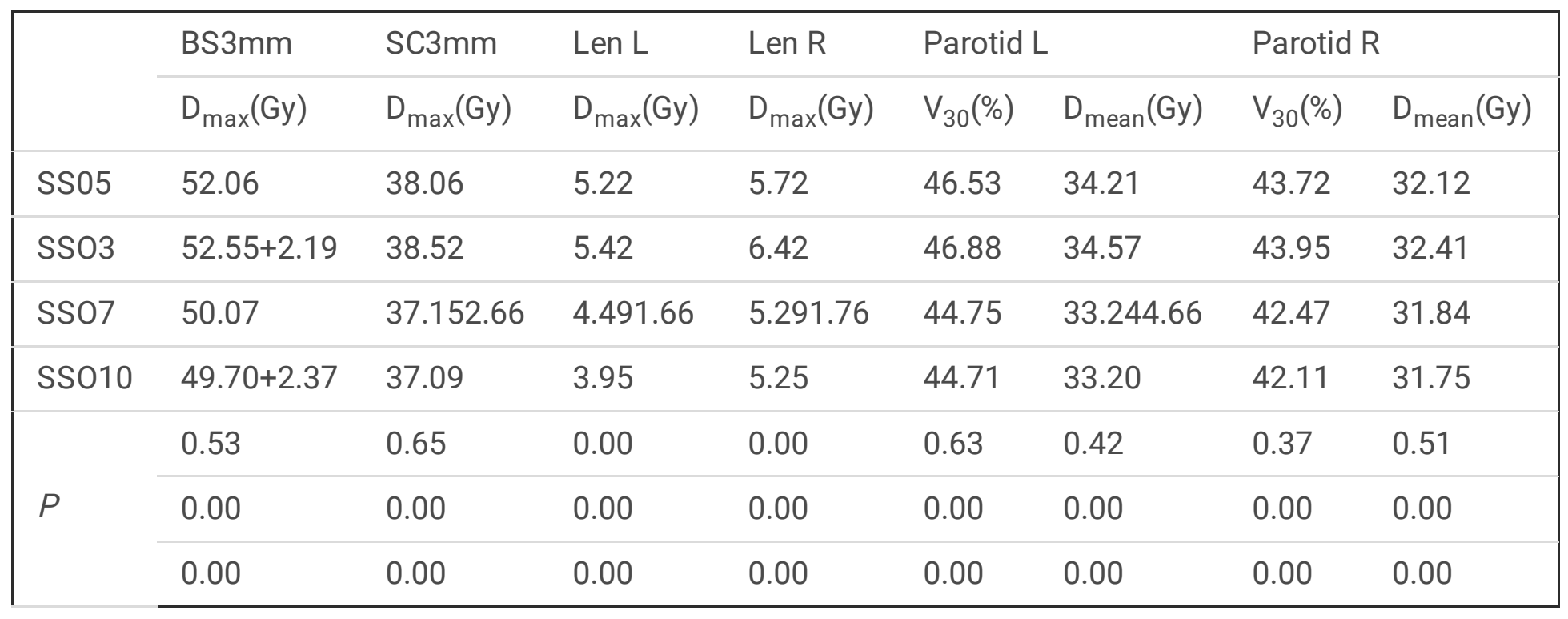

\section{Comparison of dose nephograms between groups}

In Monaco 5.4 TPS, the same layer was selected to intercept dose nephograms at the transverse, sagittal and coronal views of different SSO plans respectively, as shown in Figure 1. In the 70Gy dose nephogram distribution, there was dose spillover outside the $\mathrm{PGTV}_{70}$ target area in $\mathrm{SSO} 3$ and $\mathrm{SSO} 5$ groups, and SSO3 group was more significant than SSO5 group; in the dose nephogram distribution of 60Gy and 54Gy, dose curve gradually tightened at the three views with the increase of SSO.

Statistics and comparative analysis were performed on the prescription dose coverage rates of PGTV 70 and PTV $_{60}$ and the dose coverage rates of target areas PTV P0-54 $_{4}$ and PTV P4-54 $_{4}$ in the plan, as shown in Figure 2. There were significant differences in dose coverage rates among SSO3, SSO5 and SSO7 groups, in the meanwhile, although SSO10 group had more advantages than SSO7 group, the differences between groups had been significantly reduced.

\section{Comparison of sub-field shape between groups}

In the BEV interface, sub-field shapes of different SSO groups at $30^{\circ}, 120^{\circ}, 240^{\circ}$ and $330^{\circ}$ were captured, as shown in Figure 3. In the four different angles, the shape of the sub-field was similar, but the combined volume of the sub-field decreased with the increase of SSO, especially in the neck part.

\section{Comparison of characteristic parameters of Monaco TPS between groups}

The number of segment\#, accelerator monitor units (MU\#), optimization time (OT), and delivery time (DT) was obtained from the console window of Monaco 5.4 TPS, as shown in Figure 4. With the increase of SSO, segment\# and DT increased, while MU\# decreased, but the amplitude was very small; in contrast, OT changed significantly, the difference between adjacent groups was more than 100 s, besides, the difference between SSO3 and SSO10 groups was more than 500s.

\section{Comparison of validation results between groups}


Each SSO group plans were verified by using ArcCheck, and results were analyzed by using SNC Patient validation analysis software under $3 \%-3 \mathrm{~mm}$ Gamma ${ }^{[14]}$, as shown in Figure 5 . Results of all the plans in each group met the clinical requirements, and there was a little differenced between different SSO plans in the same group.

\section{Discussion}

XVMC algorithm is the core of Monaco TPS. The optimization processes of plan can be obtained and analyzed by the optimization console function. It is found that the whole optimization process is through several times of SSO optimization to get the final dose distribution results. Each SSO is an optimization cluster and it is similar to a large volume iterative optimization, which contains several sub-optimizations of inter loops and outer loops. The quasi - annealing algorithm is followed within each optimization cluster and the quasi - genetic algorithm is followed between clusters ${ }^{[15,16,17]}$.

In version 5.1, the SSO optimization process consists of once sub-field generation optimization (the first SSO optimization), thrice sub-field alignment optimizations (the second to the fourth SSO optimization) and once sub-field selection and merger optimization(the fifth SSO optimization). In version 5.4, the sub-field alignment optimization has been upgraded from a fixed number of 3 times to a user-defined time. It has promoted the change of planning design efficiency and plan quality.

Since each SSO optimization is equivalent to an iterative optimization of a large volume, the formula of dose influenced by the number of iterations proposed by Llacer $\mathrm{J}$ et al. in 2001 can be referred to derive the formula (2) of dose influenced by the number of SSO ${ }^{[18,19]}$. When the accumulated dose of the current beam being evaluated is closer to the accumulated dose of adjacent units, the value of the influence rate will approach 0 , and the closer it approaches 0 , the more uniform dose distribution will be. Therefore, smaller value of the influence rate is the recommended and accepted value scheme of $\mathrm{SSO}^{[20]}$.

$$
\Delta \sigma=\alpha \sum_{j}\left(D_{j}^{\left(N_{S S o}\right)}-\beta_{n} \sum_{\theta \in N_{j}} D_{\theta}^{\left(N_{s s o}\right)}\right)
$$

Note: Where $\Delta \sigma$ is the influence rate of SSO on dose; $\mathrm{a}$ is the dose filtration factor; $\mathrm{j}$ and $\theta$ is the current beam and the adjacent unit; $\beta$ is the dose receiving weight of the adjacent unit; $\mathrm{N}_{\mathrm{SSO}}$ is the optimization times of SSO; $D_{j}$ is the dose of target to be evaluated; $D_{\theta}$ is the dose of the adjacent unit.

The statistical analysis results of dose results in this study indicated that both the assessment values of the target area and the major OARs were improved to varying degrees with the increase number of SSO, that is, the difference between the dose of the evaluated grid and the dose of the adjacent grid gradually decreased with the increase in the number of SSO, and the influence rate value obtained according to formula (2) will gradually decrease; it follows that the increase in the number of SSO will promote the development of plan results towards better plan quality. In the analysis of dose nephograms at same layers, the same conclusion can be drawn. Where SSO3 has obvious 70Gy dose spillover outside the structure of PGTV 70 target area, and SSO5 has a small amount of spillover, while SSO7 and SS010 had no 70Gy dose spillover and were tightly wrapped 
around PGTV 70 . In addition, the same trend was also found in the difference comparison of dose coverage in the target area.

In the two studies conducted by Llacer J et al. in 2003 and in 2004, the number of iterations and initial intensity in intensity modulation optimization have guiding significance for the quality of the plan, however, when the number of iterations is large enough to a certain value, the plan has reached the optimum, and the plan results cannot be further optimized by continuing calculation ${ }^{[21,22]}$. On the contrary, small and high frequency changes with low eigenvalues appear, which is not conducive to the implementation of treatment plans. SSO3 SSO10 plans in this study, the results of both the target area and the major OARs, or dose nephograms at same layers, have reflected the increase of the number of SSO optimization times on improving the quality of plan, but there had no bottleneck or small and high frequency changes with low eigenvalues to SSO optimization, thus it can be seen that 10 times of SSO optimization did not reach the limit of XVMC optimization. More times of SSO optimization will help improve the quality of the plan further. However, the indicators of the target area and major OARs of SSO7 and SSO10 are pretty close, and the dose nephograms distribution is similar. Therefore, more times of SSO optimization will have less improvement on the quality of NPC plan, and there may be optimization bottleneck of small and high frequency changes with low eigenvalues. In addition, as the number of SSO optimization increased from 3 to 10 times, in the comparison of sub-field shapes at 4 same angles, the sub-field alignment area gradually decreased with the increase of the number of SSO optimization, and a few isolated island sub-fields appeared in the 10 times of SSO optimization. Therefore, in order to meet the requirements of complex NPC plans, the positioning accuracy of MLCs and the stability of gun current needed to be higher, and the emergence of isolated island sub-field would be a big challenge to the pass rate of clinical plans. However, in this study, all the ArcCheck verification results of the 3-10 times of SSO optimization met the acceptance criteria of clinical plan, and the results were similar. Therefore, the 3-10 times of SSO optimization were all suitable for the application of NPC clinical plan.

In the comparison of NPC plans with different SSO optimization times, although segment\# and DT showed an increasing trend, there was no significant difference, it could be attributed to the same restriction on the number of sub-fields for all plans in Monaco TPS parameter setting. While MU\# showed a decreasing trend, there were two main reasons. Comparison of sub-field shape at same angle, with the number of SSO optimization increased, the sub-field alignment area decreased, and the irradiation area decreased, thus resulting in the decrease of the demand for MU\#; In addition, as the number of SSO optimization increased, the calculation accuracy of the particle mass stop ratio increased, that is, the energy of per unit particle was more fully utilized, thus reducing the demand for MU\# ${ }^{[23]}$. Furthermore, it would help to decrease secondary cancer risk (SCR) in VMAT due to low doses to healthy tissues induced by scatter and leakage radiation from gantry head ${ }^{[24,25,26 \text {, }}$ 27]. The statistical results of OT showed the most obvious difference, and the difference between adjacent groups was greater than 100s. The average OT of SSO10 group was more than 1000 s, but the improvement effects of SSO10 group on the plan quality was not significantly improved compared with that of SSO7 group, and it did not get a good plan quality-speed-benefit ratio. Besides, the high number of SSO optimization required better hardware support for Monaco services, especially memory for floating-point storage and release.

\section{Conclusion}


In brief, for the clinical plan design of NPC, more SSO optimization times will help to improve the plan quality. However, under the premise of balancing efficiency of the plan design and quality of the plan, 7 times of SSO optimization are recommended.

\section{Abbreviations}

NPC: Nasopharynx carcinoma

SSO: Segment shape optimization

OAR: Organ at risk

BEV: Beam eye view

PTV: Planning target volume

TPS: Treatment planning system

XVMC: X-ray voxel Monte Carlo

VMAT: Volumetric modulated arc therapy

QUANTEC: Quantitative Analysis of Normal Tissue Effects in the Clinic

Cl: Conformity index

HI: Homogeneity index

OT: Optimization time

DT: Delivery time

MU: Monitor units

MLC: Multi-leaf collimator

SCR: secondary cancer risk

\section{Declarations}

\section{Availability of data and materials}

The datasets used and analysed during the current study are available from the corresponding author on reasonable request.

\section{Acknowledgements}

This study was supported by Grant No.20200789 from the Key Medical Research Project of Hebei Province. 


\section{Funding}

No funding has been provided for the study.

Author information

Affiliations

Department of Oncology, Xinghua People's Hospital, Taizhou 225570, Jiangsu, China

Xiaolong HUA, Jianhe YU, Qun REN, Chuanjun SONG, Wenjing XU \& Hongxia XU

Department of Radiotherapy, Anshan Cancer Hospital, Anshan 114000, Liaoning, China

Lu WANG, Li CHEN \& Yanshu MOU

Software Product Manager Room 1803, Elekta Instrument (Shanghai) Ltd, Pudong 200120, Shanghai, China Jiaqi DAl

\section{Contributions}

Xiaolong HUA set up experimental ideas and wrote papers.

Jiaqi DAl designed the experiment.

Jianhe YU and Qun REN countered the cases and reviewed them.

Li CHEN and Yanshu MOU analyzed and sorted out the experimental data.

Chuanjun SONG, Wengjing XU and Hongxia XU carried out experiments and collated data.

Lu WANG analyzed the experimental data and drafted the paper.

\section{Corresponding authors}

Correspondence to Lu WANG.

\section{Ethics declarations}

Ethics approval and consent to participate

Not applicable.

Consent for publication

Not applicable.

Competing interests 
There are no conflicts of interest in connection with this work, and the material described is not under consideration for publication elsewhere.

\section{References}

1. Yu MC. Diet and nasopharyngeal carcinoma. Prog Clin Biol Res. 1990;346:93-105.

2. Chan AT, Teo PM, Johnson PJ. Nasopharyngeal carcinoma. Ann Oncol. 2002;13:1007-15. 10.1093/annonc/mdf179.

3. Wani SQ, Khan T, Wani SY, et al. Nasopharyngeal Carcinoma: A 15Year Study with Respect to Clinicodemography and Survival Analysis[J]. Indian Journal of Otolaryngology Head Neck Surgery. 2016;68(4):1-11.

4. Ma C, Mok E, Kapur A, et al. Clinical implementation of a Monte Carlo treatment planning system[J]. Med Phys. 1999;26(10):2133-43.

5. Denis F, Garaud P, Bardet E, et al. Final Results of the 94-01 French Head and Neck Oncology and Radiotherapy Group Randomized Trial Comparing Radiotherapy Alone With Concomitant Radiochemotherapy in Advanced-Stage Oropharynx Carcinoma[J]. J Clin Oncol. 2004;22(1):69-76.

6. Shibata T. Treatment Planning of IMRT for Head and Neck Malignancies[M]. Springer Japan; 2015.

7. Teoh M, Clark CH, Wood K, Whitaker S, Nisbet A. Volumetric modulated arc therapy: a review of current literature and clinical use in practice. Br J Radiol. 2011;84(1007):967-96.

8. Srivastava SP, Cheng CW, Das IJ. The dosimetric and radiobiological impact of calculation grid size on head and neck IMRT. Pract Radiat Oncol. 2017;7(3):209-17.

9. Shanmugaratnam K, Chan SH, de-Thé G, et al. Histopathology of nasopharyngeal carcinoma: correlations with epidemiology, survival rates and other biological characteristics[J]. Cancer. 2015;44(3):1029-44.

10. Zhou X, Ou X, Xu T, Wang X, Shen C, Ding J, et al. Effect of dosimetric factors on occurrence and volume of temporal lobe necrosis following intensity modulated radiation therapy for nasopharyngeal[J]. Radiat Oncol Biol Phys. 2014;90(2):261-9. 10.1016/j.ijrobp.2014.05.036.

11. Ivo Beetz, Roel JHM, Steenbakkers O, Chouvalova, Johannes A, Langendijk, et al. The QUANTEC criteria for parotid gland dose and their efficacy to prevent moderate to severe patient-rated xerostomia. Acta oncologica (Stockholm, Sweden). 53. 10.3109/0284186X.2013.831186.

12. Teoh M, Clark $\mathrm{CH}$, Wood K, Whitaker S, Nisbet A. Volumetric modulated arc therapy: a review of current literature and clinical use in practice. Br J Radiol. 2011;84:967-96. 10.1259/bjr/22373346.

13. Rose PG, Bundy BN, Watkins EB, et al. Concurrent cisplatin-based radiotherapy and chemotherapy for locally advanced cervical cancer[J]. N Engl J Med. 1999;340(15):1144-53.

14. Fraser I. Quality assurance in radiotherapy[J]. Ir Med J. 1988;81(1):5.

15. Aubry J-F, Beaulieu F, Sévigny C, Beaulieu, Luc, Tremblay D. Multiobjective optimization with a modified simulated annealing algorithm for external beam radiotherapy treatment planning. Medical physics. 2007;33:4718-29. 10.1118/1.2390550.

16. Cotrutz $\mathrm{C}$, Lahanas $\mathrm{M}$, Kappas $\mathrm{C}$, et al. A multiobjective gradient-based dose optimization algorithm for external beam conformal radiotherapy[J]. Phys Med Biol. 2001;46(8):2161-75. 
17. Lahanas M, Schreibmann E, Baltas D. Multiobjective inverse planning for intensity modulated radiotherapy with constraint-free gradient-based optimization algorithms[J]. Phys Med Biol. 2003;48(17):2843.

18. Llacer J, Solberg TD, Promberger C. Comparative behaviour of the dynamically penalized likelihood algorithm in inverse radiation therapy planning[J]. Phys. Med. Biol. 46 2637-63.

19. Webb S. Optimizing radiation therapy inverse treatment planning using the simulated annealing technique[J]. International Journal of Imaging Systems Technology. 2010;6(1):71-9.

20. Freud N. Efficient simulated annealing for full inverse treatment planning[C]. IEEE Nuclear Science Symposium \& Medical Imaging Conference. IEEE, 2013; 10.1109/NSSMIC.2013.6829317.

21. Llacer J, Deasy JO, Portfeld TR, et al.Absence of multiple local minima effects in intensity modulated optimization.with dose-volume constraints[J]. Phys Med Biol. 2003;48(2):183-210.

22. Llacer J, Agazaryan N, Solberg TD, et al. Degeneracy,frequency response and filtering in IMRT optimization [J]. Phys Med Biol. 2004;49(13):2853-80.

23. Lu WANG, Shuangjun ZHANG, Xiaoxiao ZHAN, et al. The effect of statistical uncertainty on SBRT program in non-small cell lung cancer [J]. Cancer prevention treatment. 2020;33(1):33-40.

24. Lee B, Lee S, Sung J, Yoon M. Radiotherapy-induced secondary cancer risk for breast cancer: 3D conformal therapy versus IMRT versus VMAT. J Radiol Prot. 2014;34(2):325-31.

25. Kourinou KM, Mazonakis M, Lyraraki E, Stratakis J, Damilakis J. Scattered dose to radiosensitive organs and associated risk for cancer development from head and neck radiotherapy in pediatric patients. Phys Med. 2013;29(6):650-5.

26. Lee HF, Lan JH, Chao PJ, Ting HM, Chen HC, Hsu HC, Lee TF. Radiation-induced secondary malignancies for nasopharyngeal carcinoma: a pilot study of patients treated via IMRT or VMAT. Cancer Manag Res. 2018;10:131-41.

27. Sakthivel V, Mani GK, Mani S, Boopathy R. Radiation-induced second cancer risk from external beam photon radiotherapy for head and neck cancer: impact on in-field and out-of-field organs. Asian Pac $\mathrm{J}$ Cancer Prev. 2017;18(7):1897-903.

\section{Supplementary}

Due to technical limitations, Table 1 is only available as a download in the Supplemental Files section.

\section{Figures}



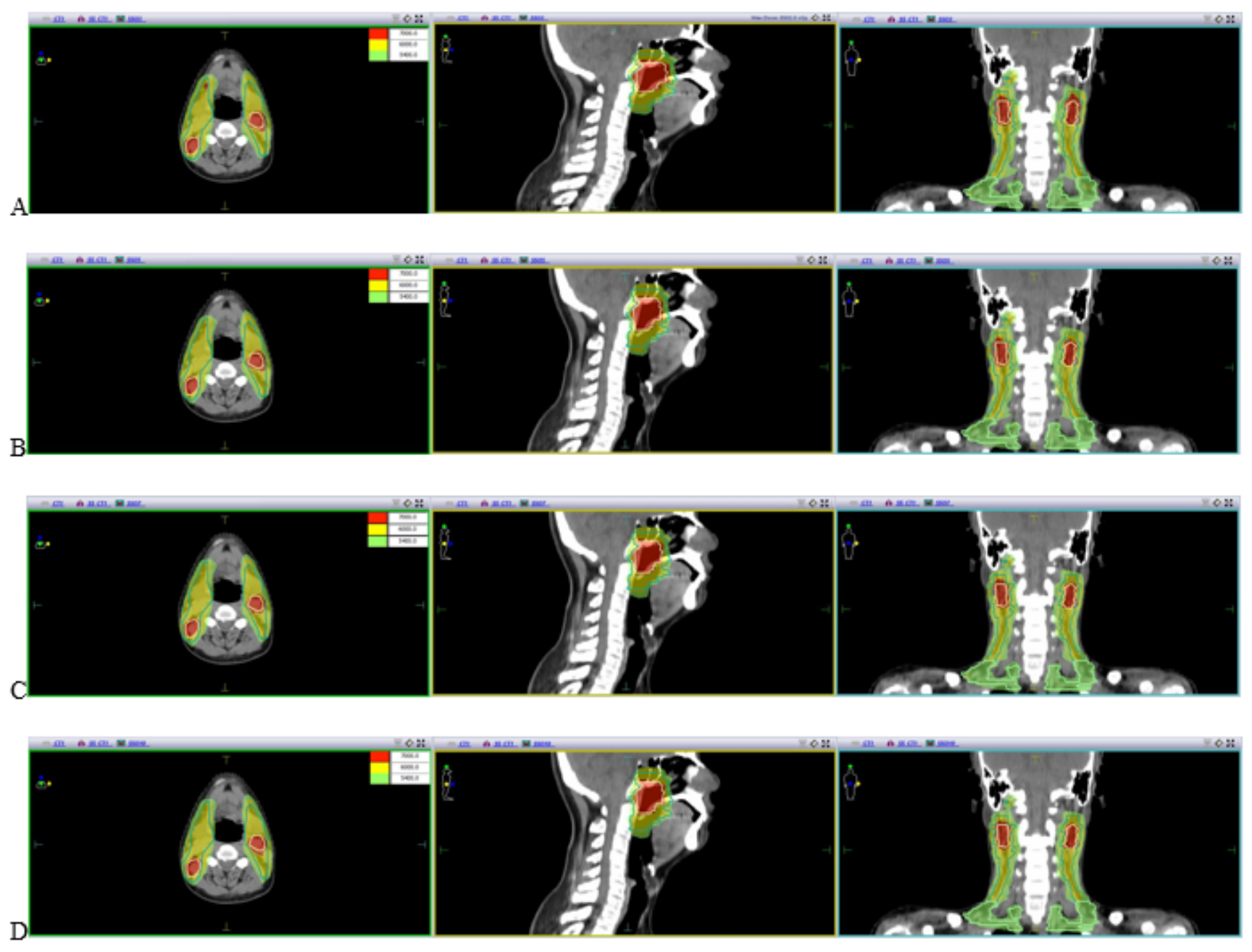

Figure 1

Color washing of different SSO plans Note: A D are SSO3 SS010 plan groups, each group is transverse, sagittal, and coronal from the left. 


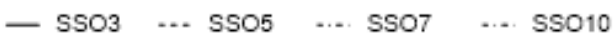

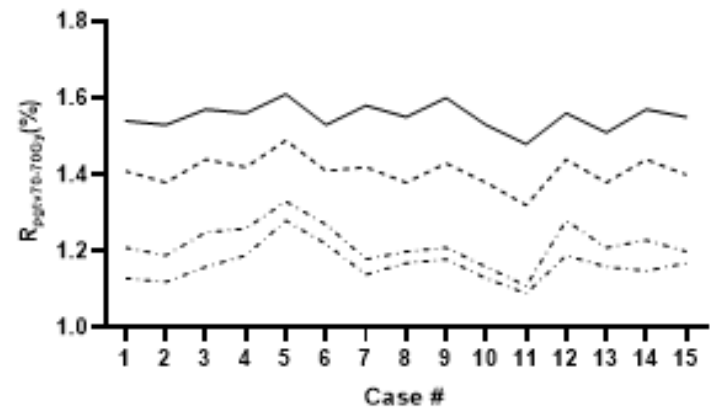

A

$\begin{array}{lllllll}-\mathrm{SSO} 3 & \ldots & \mathrm{SSO} 5 & \ldots & \mathrm{SSO} 7 & \ldots . & \mathrm{SSO} 10\end{array}$

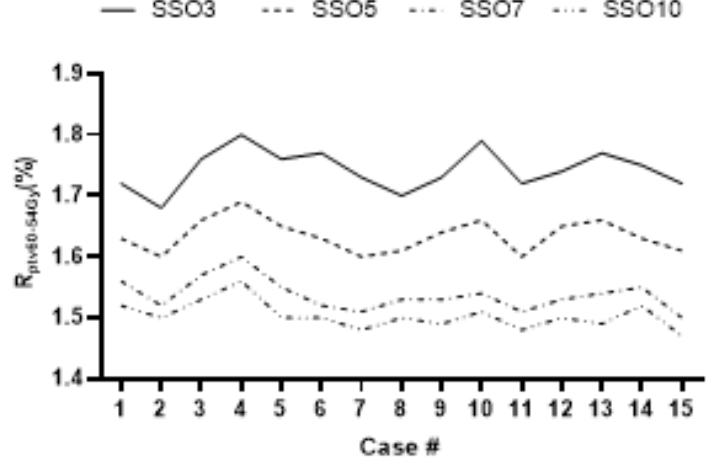

$\mathrm{C}$

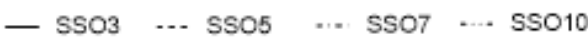

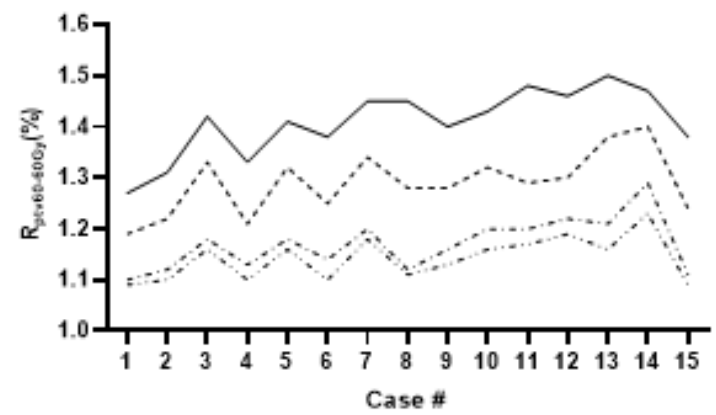

B
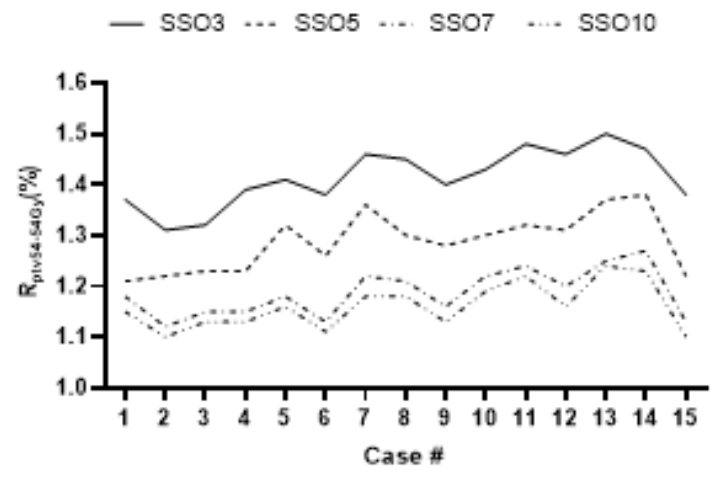

Figure 2

Dose coverage rate of different SSO
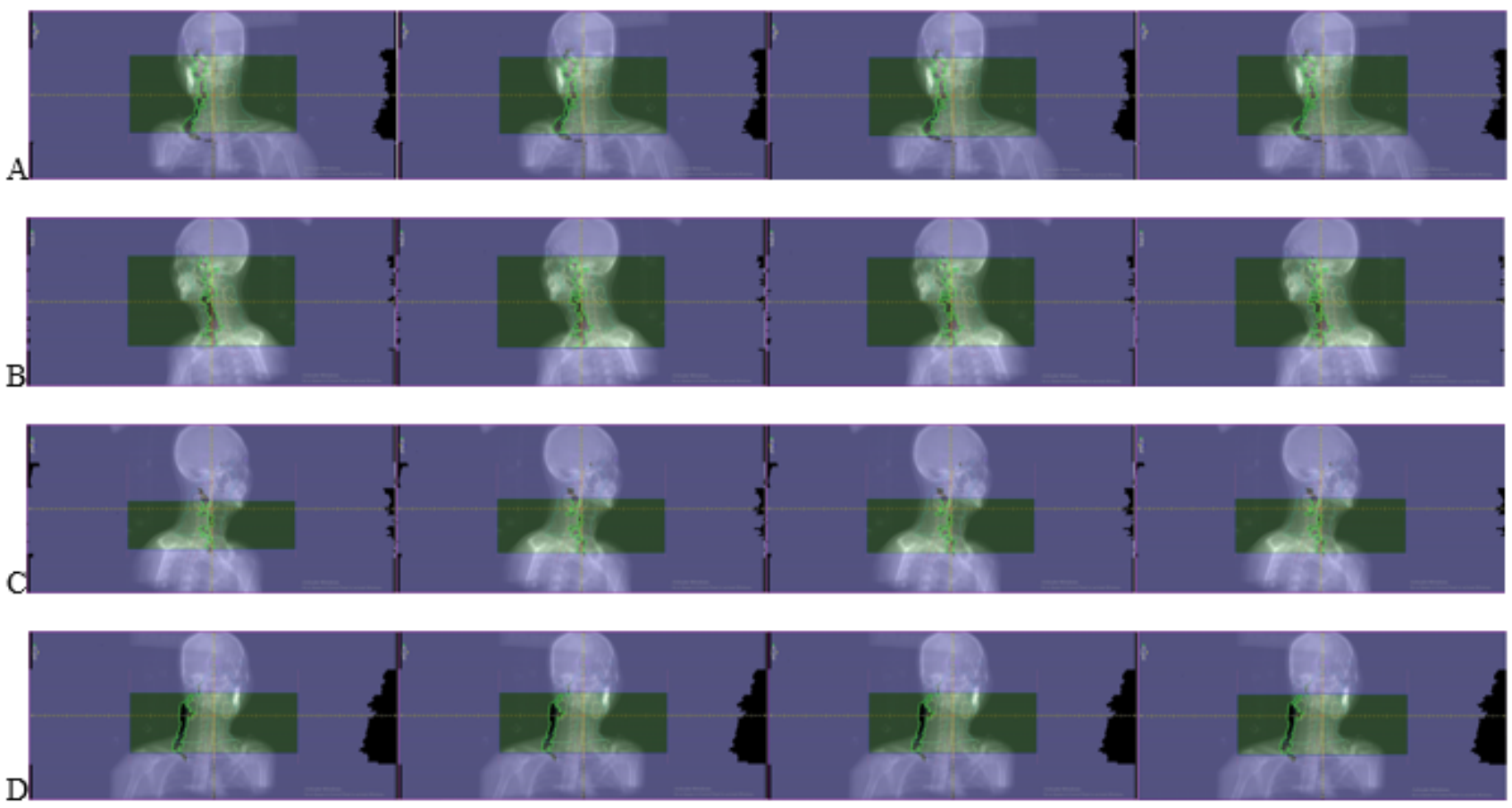

Figure 3 
Different SSO plan sub-field shape alignment Note: A D are SSO3 SSO10 plan groups, each group is $30^{\circ}, 120^{\circ}$, $240^{\circ}$ and $330^{\circ}$ from the left.

A

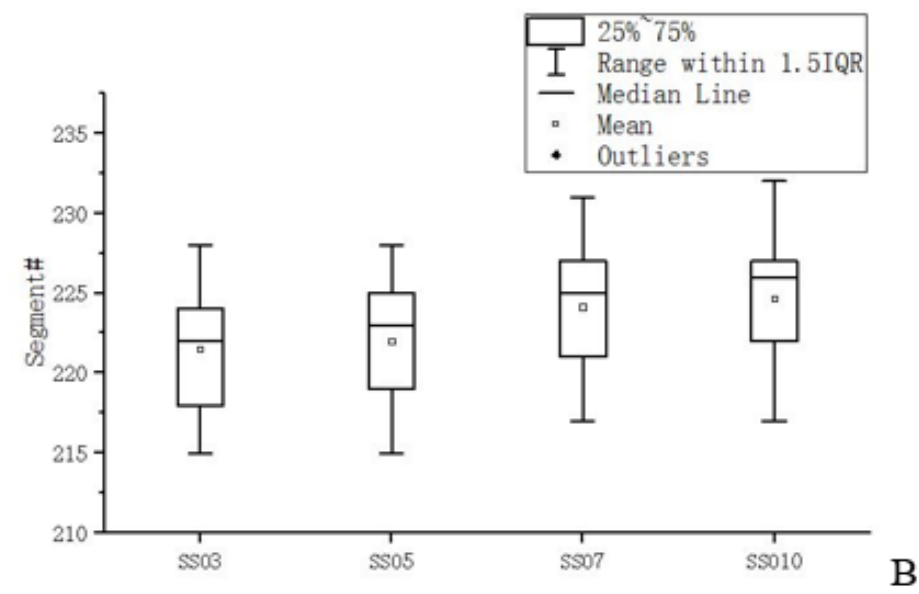

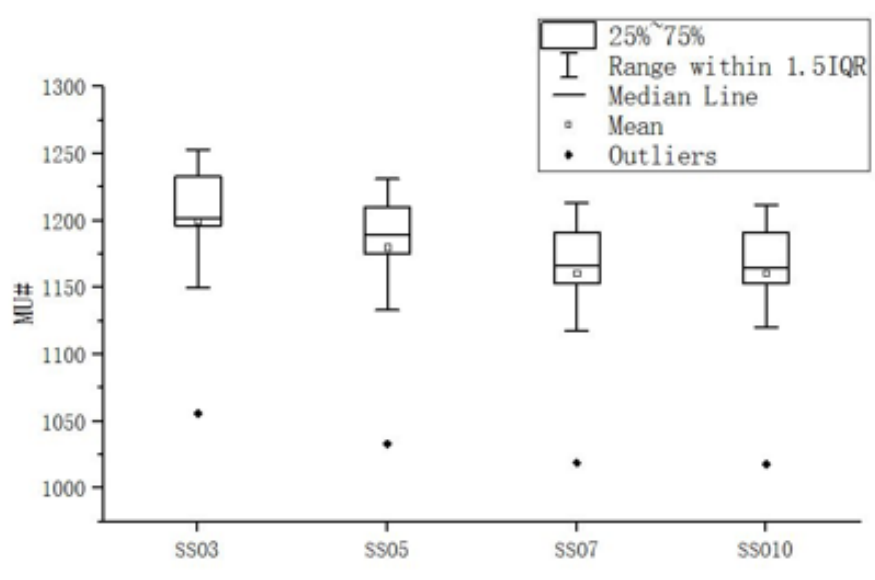

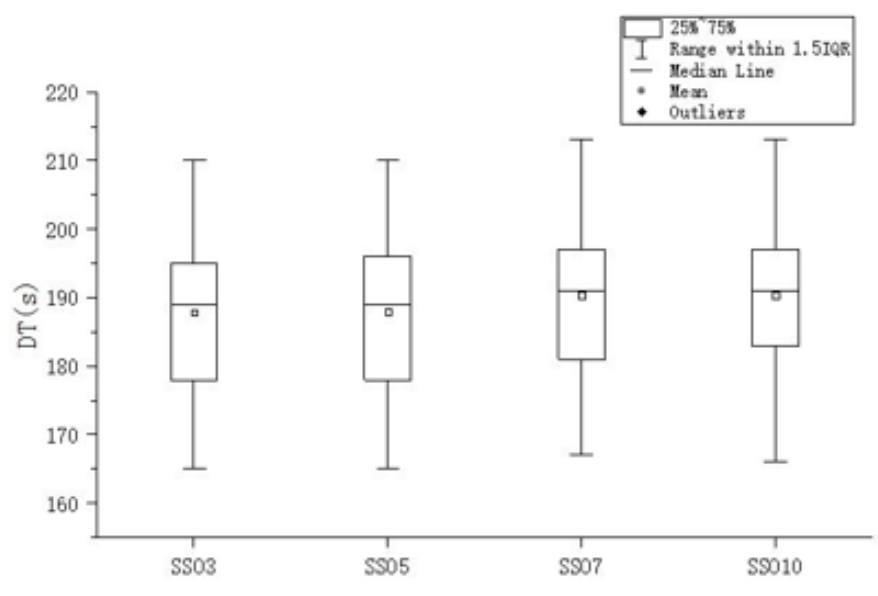

Figure 4

Comparison of XVMC parameters of different SSO 

- $\mathrm{SSO} 3$
- SSO5
$\Delta \quad$ SSO7
- SSO10

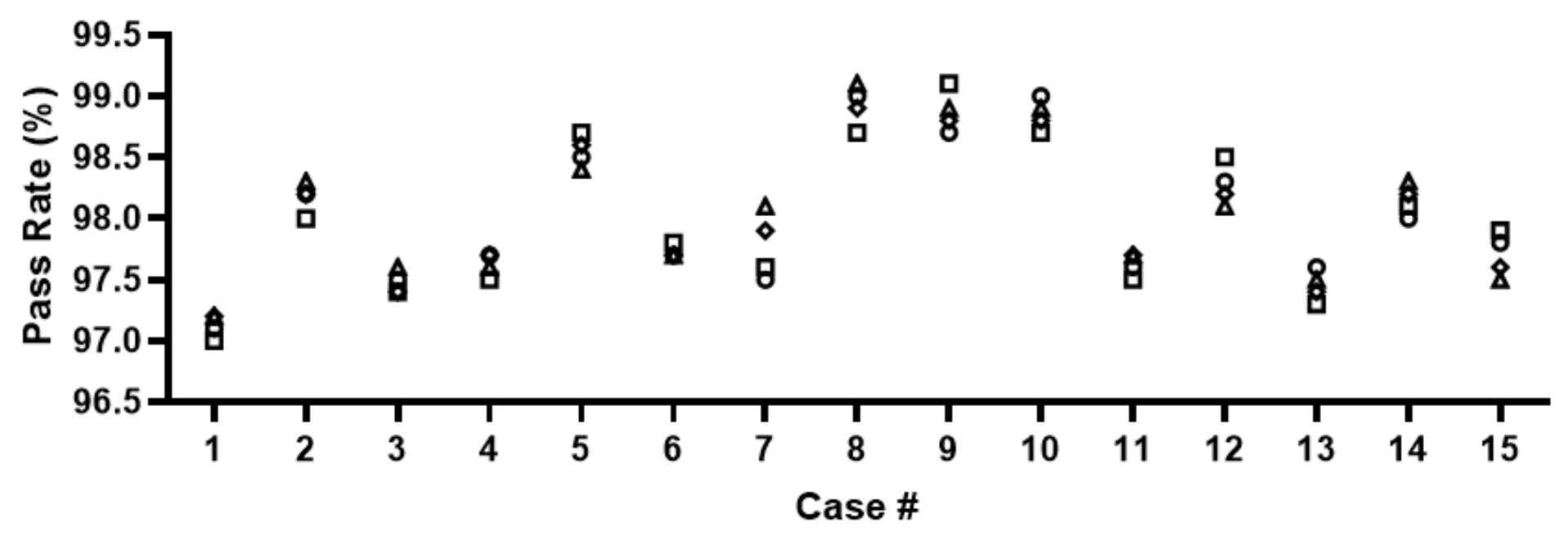

Figure 5

Comparison of verification results of different SSO.

\section{Supplementary Files}

This is a list of supplementary files associated with this preprint. Click to download.

- Table1.docx 